www.nature.com/cmi

\title{
EDITORIAL
}

\section{Viral immunology: reunion of the conjoined twins disciplines}

\author{
Chi-Chiang Yang ${ }^{1,2}$
}

\section{Cellular \& Molecular Immunology advance online publication, 4 January 2016; doi:10.1038/cmi.2015.94}

It is generally accepted that the concept of the virus as a replicative organism different from other microorganisms, and the scientific study of viruses and the infections they cause, began in the closing years of the nineteenth century. In 1884, Charles Chamberland (18511931) invented a filter-known today as the Chamberland filter-with pores smaller than the size of bacteria to completely remove bacteria from a solution. ${ }^{1}$ In 1892, Dmitry Ivanovsky (1864-1920) used Chamberland filters to show that leaf sap from a diseased tobacco plant remained infectious to healthy tobacco plants despite the filtration step. ${ }^{2}$ This result is the first evidence of the existence of viruses. Nevertheless, it was not until 1898 that Martinus Beijerinck (18511931) repeated the experiments and called the filtered infectious substance a 'virus' and not a 'toxin', and this discovery is considered to be the beginning of the field of virology. However, the definition of 'virus', an obligatory intracellular parasite smaller than bacteria containing both nucleic acid and protein that replicates by assembly but not division, was established in the 1950s, less than 60 years ago. The realization that many plant and animal diseases can be attributed to this newly recognized type of agent came even more recently. In

${ }^{1}$ Department of Clinical Laboratory, Chung Shan Medical University Hospital, Taichung 40201 and ${ }^{2}$ School of Medical Laboratory and Biotechnology, Chung Shan Medical University, Taichung 40201. Email: cyang@csmu.edu.tw

Received 5 October 2015; Accepted 5 October 2015
1796, Edward Jenner (1749-1823) developed the first smallpox vaccine to protect against viral infection, ${ }^{3}$ and his work is widely regarded as the foundation of immunology despite the fact that he was neither the first to suggest that infection with cowpox conferred specific immunity to smallpox nor the first to attempt cowpox inoculation for this purpose. Moreover, at that time, he did not know the disease etiology and did not know that viruses existed. Some scientists, however, would rather believe that this point in time was the beginning of a new discipline known as virology. It is therefore reasonable to consider these two disciplines as conjoined twins that are closely related and were birthed at the same time.

Currently, viral immunology is a rapidly growing field that has exclusively covered all aspects of human and animal viral immunology, including research and development of viral vaccines, immunological characterization of viral components, viral infection immunity, virus-based immunological diseases, immunopathogenic mechanisms, viral immunological diagnoses and methods, and immunology with tumor viruses. The interactions between viruses and the hosts they infect are multifactorial. In this special issue of Cellular and Molecular Immunology, three invited expert reviews presented the most recent advances in this field for the readers. In the first review, the complexity and functionality of the pleiotropic interferon (IFN) system in hepatitis $\mathrm{C}$ virus (HCV) infection were discussed. ${ }^{4}$ Viral

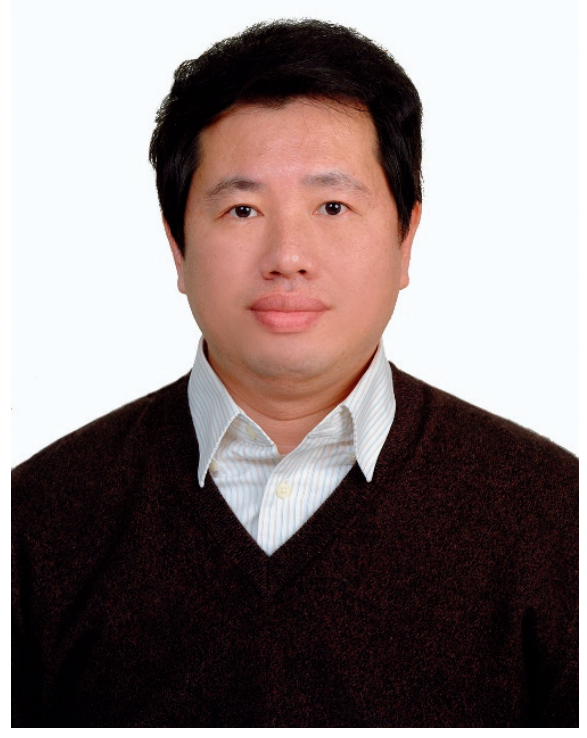

Chi-Chiang Yang

infection often triggers a first-line host defense through the production of type I IFN, which is a broadly acting antiviral cytokine, and other inflammatory cytokines. These proteins induce an antiviral state in the host cells, thereby interfering with viral replication. Wong and Chen comprehensively outline what is currently known regarding the signaling pathways involved in the production of type I IFN and IFN-stimulated genes (ISGs) and the tactics that HCV uses to subvert innate immunity. The effector mechanisms of scaffold ISGs, which were identified from genome-wide siRNA screens, known to modulate IFN function in HCV replication were summarized. The elucidation of the specificity 
and the mode of action of these emerging ISGs is useful for the identification of novel cellular targets against which effective HCV therapeutics can be developed. Wong and Chen also discussed the functions of several cellular determinants critical for regulating host immunity.

In the second review, Liu et al. reviewed the current literature on how various effectors of the immune system initiate the cytokine storm and exacerbate pathological damage in hosts with severe influenza virus infection. ${ }^{5}$ The persistent outbreaks of influenza and avian influenza in recent years suggest that influenza is a major threat to public health. In the event of a viral infection, the severity of a disease is the result of the interplay between the viral virulence and host immune resistance. Pro-inflammatory cytokines or chemokines can lead to the recruitment of inflammatory cells. When the pathogen-associated molecular patterns from the virus are recognized by the pattern recognition receptors of innate immune cells, the inflammatory response begins. ${ }^{6,7}$ Next, an increased expression of inflammatory, antiviral, and apoptotic genes occurs and is accompanied by abundant immune cell infiltration and tissue damage. As the cytokine network is important for the process of viral infection and can be potential targets for therapeutic intervention, it would be helpful to investigate the efficacy of immunomodulatory therapy in these infections. In this article, the current immunomodulatory strategies for the treatment of cytokine storms in severe influenza, including corticosteroids, peroxisome proliferator-activated receptor agonists, sphingosine-1-phosphatereceptor 1 agonists, cyclooxygenase-2 inhibitors, antioxidants, anti-TNF therapy, intravenous immunoglobulin therapy, statins, arbidol, herbs, and other potential therapeutic strategies were also reviewed.
Finally, in the third review, the protective immune responses elicited by the vaccine against yellow fever virus were discussed to provide insights for the development of a protective dengue virus (DENV) vaccine. ${ }^{8}$ As many as 400 million dengue infections occur every year, of which 96 million manifest clinically as dengue fever. ${ }^{9,10}$ Coincidentally, during the preparation of this editorial, we found that Taiwanese records show more than 20000 cases of dengue fever this year, which is the most in the country's history for this time of year. The outbreaks in other Southeast Asian countries, including Malaysia, Singapore, and Vietnam, have also been historically severe this year. The use of vaccines to prevent viral infection is the most cost-effective public health strategy. However, there are currently no vaccines to prevent infection with dengue virus. Liang et al. closely examined the immunological factors that have been associated with the protection conferred by the yellow fever virus (YF17D) vaccine in the hope of establishing a strategy for the development of an efficacious DENV vaccine. The kinetics of various types of the immune response, including the efficient activation of APC, the induction of potent innate immune responses, the production of pro-inflammatory cytokines, the eliciting of high titers of neutralizing antibodies, the induction of poly-functional CD4+ and CD8+ $\mathrm{T}$ cells, and the stimulation of long-term cellular memory and humoral immunity induced by the YD-17D vaccine were discussed in detail. Hopefully, a careful examination of the immune responses to DENV infection, and a comparison of that with the knowledge on YF infection and YF17D vaccine-elicited protection, may help to establish a strategy for the development of a dengue vaccine.

Virus infection can result in acute or chronic disease, which represents a con- tinuing threat to human health. In the past, we have achieved some notable successes in the struggle against viruses, e.g., the eradication of smallpox in the 1980s. However, the success of smallpox eradication has made a large fraction of the population now susceptible to smallpox, as mandatory smallpox vaccination was discontinued in the USA in the early 1970s. To counter the continuing threat posed by viruses, it is essential to understand the body's innate and adaptive immune responses against viral infection.

1 Teri Shors Understanding Viruses. Sudbury, MA: Jones \& Bartlett Publishers, 2008: 76-77.

2 Sussman M, Topley WWC, Wilson GK, Collier LH, Balows A. Topley \& Wilson's Microbiology and Microbial Infections. London: Arnold, 1998: 3.

3 Baxby D. "Jenner, Edward (1749-1823)". Oxford Dictionary of National Biography. Oxford University Press. Retrieved 14 February 2014

4 Wong MT, Chen SS-L. Emerging roles of interferon-stimulated genes in the innate immune response to hepatitis $\mathrm{C}$ virus infection. Cell Mol Immunol 2015; 12: 11-35.

5 Liu Q, Zhou YH, Yang ZQ. The cytokine storm of severe influenza and development of immunomodulatory therapy. Cell Mol Immunol 2015; 12: 3-10

6 Medzhitov R. Recognition of microorganisms and activation of the immune response. Nature 2007; 449: 819-826.

7 Barton GM. A calculated response: control of inflammation by the innate immune system. J Clin Invest 2008; 118: 413-420.

8 Liang HB, Lee M, Jin X, Guiding dengue vaccine development using knowledge gained from the success of the yellow fever vaccine. Cell Mol Immunol2015; 12: 36-46.

9 Bhatt S, Gething PW, Brady OJ, Messina JP, Farlow AW, Moyes CL et al. The global distribution and burden of dengue. Nature 2013; 496: 504-507.

10 Kramer LD, Ebel GD. Dynamics of flavivirus infection in mosquitoes. Adv Virus Res 2003; 60: 187-232. 